\title{
Digital urban ambience: Mediating context on mobile devices in a city
}

\author{
Jesper Kjeldskov*, Mikael B. Skov, Glen W. Nielsen, Søren Thorup, Michael Vestergaard \\ Centre for Socio-Interactive Design, Department of Computer Science, Aalborg University, Selma Lagerlöfs Vej 300,9220 Alborg East, Denmark
}

\section{A R T I C L E I N F O}

\section{Article history:}

Available online $\mathrm{xxxx}$

Keywords:

Digital urban ambience

Context-awareness

Mobile computing

Field study

\begin{abstract}
A B S T R A C T
We explore the term digital urban ambience to describe the user experience of mobile devices mediating context in metropolitan areas. Mediated context-awareness explores a constructivist approach to context and aims to provide resources for experiencing context rather than correctly recognizing and adapting to it. Exploring this approach we have built a prototype system, eSpective ${ }_{2}$, combining maps and augmented reality on a touch phone, and we have studied its use. We conducted a triangulated field study involving 58 participants and up to one month of use. Elaborating on the work of Thibaud (2002) [1], the main finding is that context resources provided through mobile devices digitally contribute to the experience or feeling of urban ambience (1) by adding to the quality of the situation as a whole, (2) as a catalyst for action, and (3) as a sensory background.
\end{abstract}

(C) 2012 Elsevier B.V. All rights reserved.

\section{Introduction}

Modern mobile phones are graphically powerful devices with a range of sensors including GPS, accelerometers, and a compass that facilitate the creation of rich and engaging interaction design and user experiences. One of the popular types of applications for IOS and Android OS is location-based services that provide rich and interactive digital information about people's immediate physical surroundings. These services and applications are no longer merely thought of as guides for tourists but more as tools for the inhabitants or visitors of towns and cities. They provide information about contextual resources, such as people and places, for finding out who is around, what to do, and where to go [2,3], or public transport [4]. Common for these is that they represent a different approach to the idea of "context-awareness" than the one dominating the field for over a decade, since it was first introduced by Schilit and Theimer [5]. Apart from using the user's location as an anchor point for interaction, these new applications do not seek to formalize and adapt automatically to their surroundings, but aim instead at mediating contextual information resources, thereby allowing users to obtain and maintain awareness of their context through interpretation. Judged by user uptake and impact on commercial applications, this approach appears to have merit.

From a human-computer interaction (CHI) perspective, however, little is known about the user experience of such "mediated" context-awareness. This has motivated us to explore the design and use of such systems. Inspired by the 2008 CHI workshop on Urban Mixed Realities [6], we have focused specifically on an urban environment. The purpose of our work has been to better understand how different aspects of urban context can be represented, how people perceive and use such context representations, and how the user experience of mobile mediated context-awareness can be described conceptually in urban settings. Understanding this, we believe, will eventually enable us to also begin to understand why it is interesting for local residents of a city to explore it through a digital mobile system, not just tourists.

The paper is structured as follows. First, we introduce related work within context-awareness. We then introduce the eSpective $_{2}$ system by describing how its interaction design supports people exploring context information in their

\footnotetext{
* Corresponding author. Tel.: +45 9949 8921; fax: +45 99409798.

E-mail addresses: jesper@cs.aau.dk (J. Kjeldskov), dubois@cs.aau.dk (M.B. Skov), glenwn@gmail.com (G.W. Nielsen), thorup@gmail.com (S. Thorup), michael@vestergaard.dk (M. Vestergaard).
} 
immediate surroundings and by outlining three different types of context information included in the system. Third, we describe a field study with quantitative and qualitative findings, and discuss themes identified across data sources. Finally, we discuss the user experience of eSpective ${ }_{2}$ as one characterized by being a part of the experience of the city. We promote the term digital urban ambience inspired by the socio-architectural notion of urban ambience to describe the specific aspect of urban ambience created by digital systems and information technology.

\section{Background and related work}

\subsection{Two views on context}

In his seminal paper "What We Talk About When We Talk About Context", Dourish [7] presents two views on context grounded in positivist and phenomenological reasoning. Following on from this, Oulasvirta et al. [8] argue that what constitutes context can be divided into two disparate camps of thought depending on their philosophical foundation: realism or constructivism. Realism has its roots in natural sciences, and believes that context is real, transcendent, and exists out there independently of any type of observer. Hence, context can be perceived by computer systems just as well as by people, and if recognized correctly it can be used to automatically adapt the behavior of systems to fit appropriately. In contrast, constructivism has its roots in human and social sciences, and believes that context is "socially constructed in interaction with other agents in the world" [8], and interpreted psychologically by human individuals and within a certain frame of reference. Hence, computers are not able to perceive and interpret context equally to the way it is understood by humans, but may, at best, be able to "provide resources for people themselves to create and maintain contexts in their action" [8]. As pointed out in [8], Oulasvirta et al.'s concept of realism maps loosely to Dourish' concept of positivism. However, the concept of constructivism defines a wider scope than the phenomenological approach promoted by Dourish. For a more elaborated discussion on the differences between the two views of context put forward by Dourish and Oulasvirta et al., see [8].

Driven by realizing the visions of Weiser [9], the literature is dominated by reports on mobile systems capable of adapting content to their context as sensed by a computer. These systems represent a realism-informed approach to context, and generally promote context-based machine intelligence as a solution to various challenges of mobile human-computer interaction such as limited screen real estate, input capabilities, and dynamic situations of use. Chen and Kotz refer to the same class of systems as active context-awareness [10], and from a realism perspective this approach is "the most interesting one" because it "leads to new applications on mobile devices", "requires more infrastructure support", and "help to eliminate unnecessary user cooperation". We label this class of systems adaptive context-awareness, but it is striking that only very little adaptive context-awareness has actually made it into the design of commercial mobile devices and systems.

From an interaction design point of view, it appears that context-based automation needs to be kept extremely minimal in order to work well, such as maps zooming in on the user's location and displays changing orientation depending on how they are held. As argued by Brown and Randell [11], one of the reasons for this is that the seemingly attractive idea of contextawareness in fact hides a large degree of complexity, and, in a more recent study, Dearman et al. conclude that considerations of context-aware automation are significantly more nuanced than originally conceived [12]. In practice, even applications that appear to be very simple, such as the mobile phone that only rings when appropriate, are highly complicated to realize because of the fundamentally different meanings and interpretations of context by humans and by machines.

Emerging context-aware systems increasingly consider that context information may itself be the content, rather than merely a filter for other information. Examples include Paulos and Goodman visualizing "familiar strangers" based on patterns of nearby Bluetooth devices [13], Erickson et al. visualizing people and their activities through "social proxies" [14], Ferris et al. [15] providing real-time travel information for bus riders, and other mobile guide systems that do not impose suggestions on the user based on context but merely offer information resources for the user to chose from [3,16,2]. These systems represent a constructivism-informed approach to context. They do not assume that it is possible or desirable to provide context-based automation, but advocate that complex and ambiguous context information is better dealt with by actively involving the human user [17-19]. While computational systems are good at gathering and aggregating data, humans are highly capable of interpreting and integrating information representations, and determining appropriate actions [20]. Making humans, and not machines, the consumers of context information by mediating it in the interface therefore appears like an attractive alternative road ahead. We label this class of systems mediated context-awareness.

In this study we have deliberately explored a constructivist approach to context. We subscribe to the view that context is a psychologically interpreted, socially constructed entity too complex to model in a computational system in its entirety, making only very simple context-based adaptation a feasible principle for mobile human-computer interaction. Informed by this view, we have investigated the design and use of mediated context-awareness, seeking to enhance user experiences by offering context information on mobile devices for people to explore and experience alongside the experience of their physical surroundings.

\subsection{Mobile systems representing context}

Several commercial systems, and some research prototypes, have explored mediated context-awareness by offering different types of context information on mobile devices, typically using the user's location as an anchor point. These systems 
can be divided into either map-based or augmented reality-based systems, depending on the way the digital information is related to the user's physical surroundings. Currently, map-based systems are the predominant approach, but with the growth of graphically powerful mobile devices with high-quality video cameras, augmented reality on mobile devices is a growing trend.

In an early research study, Fithian et al. [21] presented a mobile context-aware planning application representing the location of nearby friends and places on a map alongside functionalities for planning and coordinating meetings and events. Based on people's perception of what meaningfully constitutes a "place" in such systems, Zhou et al. [22] investigated how humanly meaningful place representations can be derived from less understandable context information such as latitude and longitude. Likewise informing the creation of meaningful context-information for human perception, Lemmelä and Korhonen [16] presented a prototype map application in which the nature of different areas of a city are visualized based on clustering of keywords, such as "restaurant" or "shopping". Exploring the use of such visualizations of context in the city, Just-for-Us was a context-aware mobile web site augmenting a city precinct with social and physical context information for people socializing out on the town [23].

As an example of a commercial product, Loopt [2] offers a suite of mediated context-aware mobile applications for the iPhone and iPad allowing users to find out who is around, what is happening, what to do, and where to go. The applications make use of map-based representations of the user's immediate surroundings overlaid with information about places, friends, events, and other people. Hence, the application suite is highly aware of spatial, physical, temporal and social context, but it allows users to explore these rather than prescribing their actions. Another commercial product is OneBusAway [4], reported by Ferris et al. [15]. This application knows about real-time arrival information for the public transport system in Seattle and lets users explore different map-based and list-based graphical representations of this context information on their mobile devices.

Exploring augmented reality (AR) as an interface approach to mediated context-awareness, Layar [3] is an AR browser for iPhone and Android use that allows users to experience their immediate surroundings by displaying digital context "layers" on top of the physical world as seen through the mobile phone camera. Combining maps and augmented reality, MapLens [24] uses a camera phone to augment a physical map with a digital overlay.

\section{The system}

Inspired by these systems and studies, and our own previous experiences with a system called eSpective [25], we designed and built a system called eSpective ${ }_{2}$ for touch phones running Android OS. eSpective ${ }_{2}$ provides users with context information related to the city, which would otherwise be invisible or difficult to see, and with interaction techniques for exploring this context information using the phone's built-in GPS, camera, compass, accelerometer, and touch screen. The basic concept is to provide a context-aware mobile application that provides resources for experiencing and interpreting context rather than one that tries to correctly recognize and adapt to it. The application presents users with a map or augmented reality view showing their current location and overlaid with information related to their immediately surrounding context. Users can explore their context in two ways.

(1) Using the touch interface to apply and adjust different "Lenses" to the map or AR view, thereby revealing and filtering different types of context information.

(2) Using the phone's motion sensor to "ArcZoom" between the map and the augmented reality view of their surroundings, thereby changing the visual range and detail.

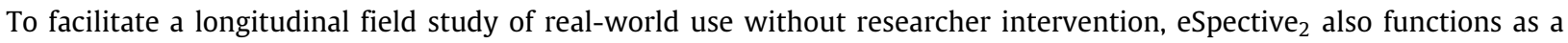
technology probe [26], logging user interaction and relaying it to the research team over the Internet.

\subsection{Lenses}

The first interaction technique in eSpective ${ }_{2}$ allowing users to explore their context is called Lenses. Lenses consist of an animated overlay of touch controls that is activated by touching the screen (Fig. 2). Lenses consist of two wheels respectively controlling the zoom level of the map and the type of information overlay. The third control, a horizontal slider, is used to fine-tune the content displayed, such as, for example, highlighting dining over drinking when presented with places for going out to.

The Lenses aim to support a playful and explorative user experience, where context information can be browsed, explored, and discovered as an interactive and entertaining activity, as opposed to a strongly goal-oriented experience, or where the system prescribes "appropriate" choices.

\subsection{ArcZoom}

The second interaction technique in eSpective ${ }_{2}$ that facilitates interactive exploration of the user's context is called ArcZoom. ArcZoom makes use of the accelerometer and compass built in to many modern mobile phones to create a smooth and seamless transition between a two-dimensional (2D) map view and an augmented reality view. This is done technically 


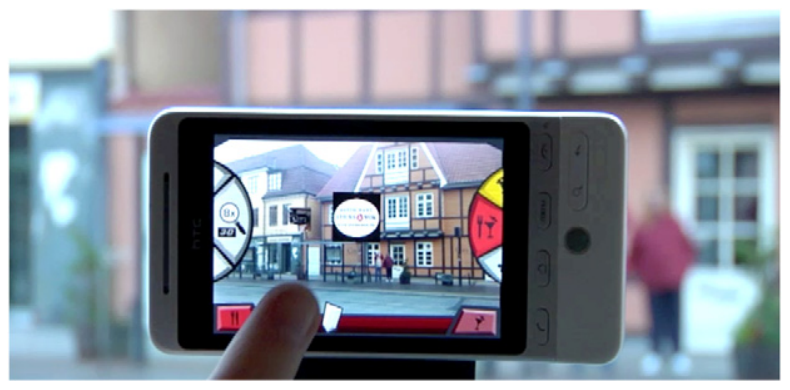

Fig. 1. Mediated context-awareness in eSpective2.

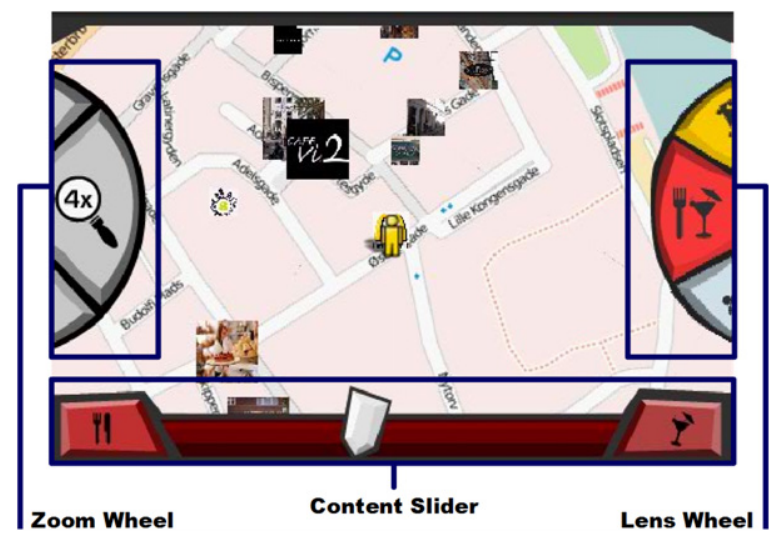

Fig. 2. Touch controls for Lenses.

by tilting and zooming within an intermediate three-dimensional (3D) map, and by blending in and out 3D graphics and the signal from the video camera. This creates an effect similar to moving a film camera in an arc from a bird's-eye view to a first-person view while simultaneously zooming.

When the phone is held horizontally, a 2D map is shown from a bird's-eye perspective (Fig. 3(A)). This is similar to holding a paper map. When tilting the phone gradually upwards, the perspective of the map tilts correspondingly towards a thirdperson perspective onto a 3D map along the direction the user is facing in the physical world, with overlaid icons standing out from the map (Fig. 3(B)). When tilting the phone further upwards, the 3D map is gradually faded out, and the live view from the built-in video camera is faded in as background with superimposed information icons. This creates an augmented reality view of the user's surroundings with graphics hovering in the air in front of the location or place they are related to (Fig. 3(C)).

Compared to other mobile systems both incorporating map and AR modes, ArcZoom's seamless and smooth transition views make the user experience appear "modeless".

\subsection{Places, people and public transport}

Exploring the application of mediated context-awareness, eSpective ${ }_{2}$ was designed to contain information about nearby places, people, and public transport. Hence the system combines the content of Loopt [2], OneBusAway [15,4], and Just-forUs [23]. While this does, of course, not cover all types of possibly relevant information content, it was deemed suitable as a starting point for investigating the use of mediated mobile context-awareness, as it involves four dimensions of context often highlighted as important for context-aware systems in the literature: spatial, social, physical, and temporal context.

\subsubsection{Places}

The Places Lens offers a representation of physical-spatial context information to the user. It provides information about physical places such as restaurants, cafés, and bars in the user's spatial vicinity, and has information about more than a hundred places of this kind within the city of Aalborg. These are each displayed on the map as a small icon showing their logo or a visually prominent aspect of their physical facade (Fig. 4). In the AR view, the icons appear to hover in the air in front of the places they relate to, as illustrated in Fig. 1.

Tapping on an icon reveals detailed information from the web about that place, such as menus, specials, opening hours, and user ratings, as well as photos taken from inside the place. This makes it possible to gauge the look and type of a place before actually entering it, and to compare them with each other. Using the Places Lens, the content slider at the bottom 


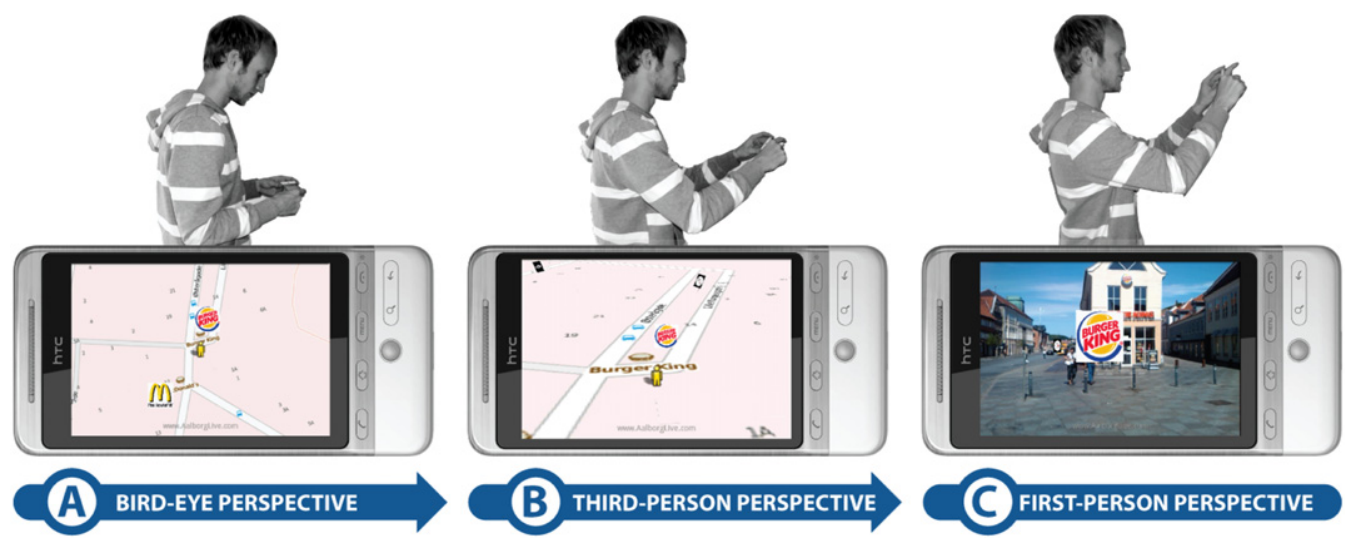

Fig. 3. Three stages of ArcZoom.

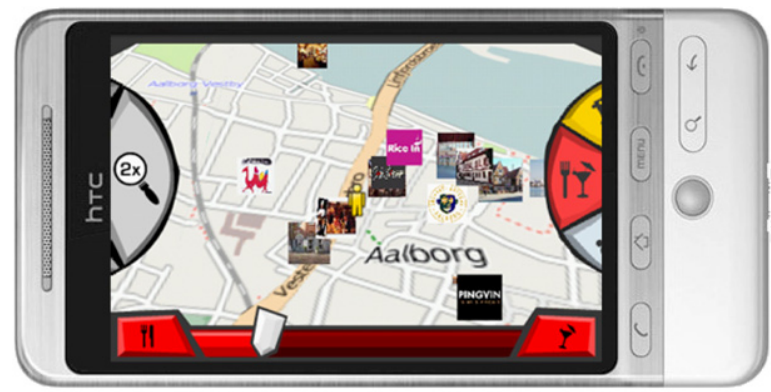

Fig. 4. 3D map view while using the Places Lens and with touch controls active.

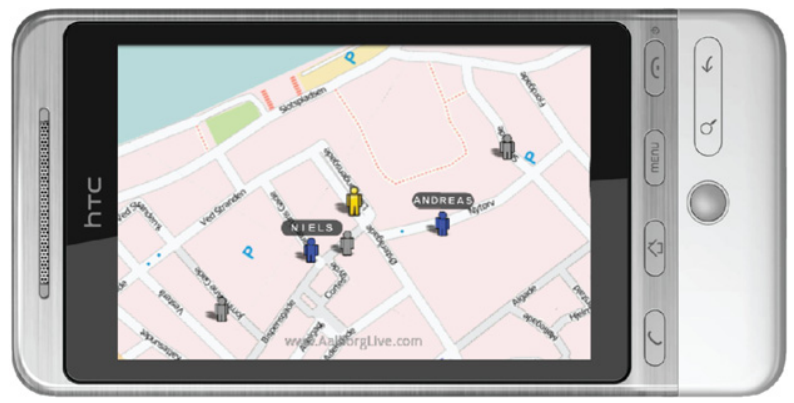

Fig. 5. 2D map view while using the People Lens.

of the screen makes it possible for the user to filter places by type. Moving the slider all the way to the left will emphasize places that mainly serve food. Moving it all the way to the right will emphasize places like bars. Placing the slider right in the middle will emphasize places like cafés, which, for example, serve as a restaurant during the day and as a bar during the night. Apart from reducing the number of places presented on the screen at the same time, the idea of the content slider is to facilitate playful interaction and exploration. Context information about places in mobile guides can easily appear static and "pre-recorded", and hence potentially boring and lifeless. By facilitating interaction opportunities that is more direct and responsive to user input (touch controls, accelerometer, compass, and AR) the aim is to create a more engaging and "live" user experience with such content.

\subsubsection{People}

The People Lens offers a representation of social-spatial context information to the user. It makes it possible to gauge social activity in the city, see the whereabouts of one's friends, and to communicate with them through the mobile phone. Each user has a list of friends, showing those nearby on the map as a blue person with a nametag (Fig. 5). It is also possible to see other users who are not one's friends. These are shown anonymously on the map in grey. Using the People Lens, the content slider can be used to filter between friends and anonymous other people. The locations are updated every $5 \mathrm{~s}$, making it possible to see them move through the city. 


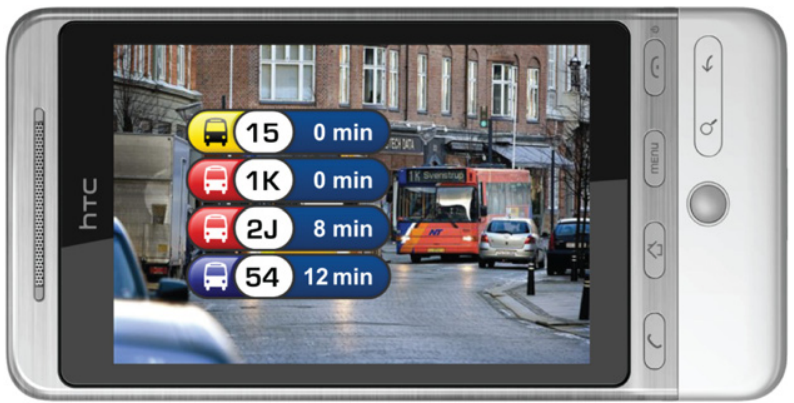

Fig. 6. AR view while using the Public Transport Lens.

By tapping on a friend's icon on the screen, a profile page will show up with options for communicating through SMS, chat, or phone call. When using the People Lens in the AR view, the user can pan the device around his/her surroundings and scout for friends or other people whose icons will appear on the screen in the direction towards them. The idea of displaying friends and anonymous people's whereabouts in the city is to use this as a contextual indicator of social activity in particular areas or locations. This, of course, requires a critical mass of users anonymously and continuously posting their locations. In order to somewhat overcome this issue for the purpose of the field studies, fictive anonymous people were programmed to appear moving around the city.

\subsubsection{Public Transport}

The Public Transport Lens offers a representation of temporal-spatial context information to the user. Similar to OneBusAway [15,4], it provides real-time information about the arrival of buses at nearby stops. Looking at the map, the locations of bus stops are indicated with icons, which also function as links to a list with information about the next ten buses arriving there. In the AR view a virtual sign with arrival information for the next four buses is shown hovering over the locations of bus stops (Fig. 6).

eSpective $_{2}$ contains live information of all 390 bus stops in the city. Arrival information is retrieved from the bus company's mobile webpage.

\subsection{Technical implementation}

eSpective $_{2}$ was implemented for the Android platform, taking advantage of its well-documented development environment and APIs allowing, for example, easy access to hardware sensor readings and hardware accelerated 3D graphics. Most of the graphical user interface was implemented using a 3D engine based on OpenGL ES. Hence, screen elements such as the map and the icons are all 3D objects in a scene handled by the 3D engine. This creates a very smooth transition, when shifting from the 2D map to the 3D map, and from the 3D map to the AR view. User interface elements such as the Zoom and Lens wheels and the content slider are animated 2D elements drawn on top of the 3D view.

The average performance of the system is 20 frames per second. Despite hardware accelerated 3D graphics, to ensure this frame rate it was necessary to limit the maximum number of icons in the 3D scene to 20 . To make the 3D engine work smoothly when using content streamed from the web, a dynamic texture loader was implemented to handle possible delays or connection losses. The map representation is based on OpenStreetMap [27], which is a free editable map that everyone can contribute to and use.

\section{Field study of use}

We studied the use of eSpective 2 in a field study using a triangulation of three different methods. According to Bogdan and Biklen [28], triangulation is a powerful approach that facilitates validation of data through cross-verification from more than two sources. Specifically, eSpective ${ }_{2}$ was studied with different types of participant, in different places, and for durations of an hour, a week, and a month, corresponding to what Denzin [29] describes as data triangulation involving different people, space, and time (see Table 1 ).

Using triangulation, we were able to obtain a more complete and nuanced picture of the system in use, including data from people who used the system over longer periods of time with and without researcher intervention, as well as data detailing actual user interaction.

\subsection{Participants}

The field study involved a total of 58 participants. The one-hour studies involved ten participants aged 22-28 and equally balanced between males and females. Each study involved 2-3 people, who knew each other well, each provided 
Table 1

Overview of triangulated field study method.

\begin{tabular}{llll}
\hline Duration & Enquiry method & Collected data & Users \\
\hline $1 \mathrm{~h}$ & Think-aloud during use & Video of use & 10 \\
& Semi-structured group interview & Audio interview & \\
1 week & $\begin{array}{l}\text { Semi-structured group interview } \\
\text { Technology probe }\end{array}$ & $\begin{array}{l}\text { Audio interview } \\
\text { System data log }\end{array}$ & 6 \\
\multirow{2}{*}{ month } & $\begin{array}{l}\text { Semi-structured phone interview } \\
\text { Technology probe }\end{array}$ & $\begin{array}{l}\text { Audio interview } \\
\text { System data log }\end{array}$ & \\
\hline
\end{tabular}

with a phone and roaming around the city centre accompanied by a researcher. The participants were recruited through noticeboards and by word of mouth.

The one-week studies involved six participants aged 21-27, five of which were males. Each study involved a group of three people, who knew each other well, freely using the system on a provided phone as a part of their daily life. The participants were recruited through noticeboards and by word of mouth.

The one-month studies involved 42 participants aged 20-26 and all male. All participants already owned and used an Android Phone as their personal mobile device, and had downloaded the eSpective ${ }_{2}$ application from the Android Market. More than 300 people downloaded the application. From this cohort of potential participants, 42 people were recruited via email based on the requirements that they (1) lived, worked, or studied in the city of Aalborg, (2) used the application more than once, and (3) were willing to participate in the study. From the 42 participants, four were recruited for qualitative follow-up interviews, based on interest in the study.

\subsection{Procedure and data collection}

The one-hour field study was conducted in the city centre of Aalborg. The participants were provided with an Android phone, each with eSpective ${ }_{2}$ preinstalled, and were given a brief introduction to the study and the eSpective ${ }_{2}$ application. They were then asked to each complete 11 tasks using the system while roaming around freely in the city centre for approximately an hour. The tasks were designed to prompt the user to explore the physical city as well as the digital context information provided in the system. Video data showing the use of the system in situ was recorded for each participant by an accompanying observer. The observer encouraged the user to think aloud, verbalizing his or her interactions with and perception of the system. After each study, a group interview was performed with all participants and researchers.

In the one-week field study the groups of participants were concurrently provided with an Android phone, each with eSpective $_{2}$ preinstalled. They were given a brief introduction to the study and the eSpective ${ }_{2}$ application, but were not asked to use it in any particularly way other than what they felt like. Throughout the week of use, interaction was continuously logged by the system and relayed to the research team. Data logs were analysed during the week of study in order to provide input to the interviews with the participants. Interviews were conducted at the end of the week when the phones were collected.

For the one-month field study, eSpective ${ }_{2}$ was made publicly available for download from Android Market. To download the application one just had to have an Android touch phone and a Google account. In order to advertise the application, posters with information and download instructions were distributed throughout the university campus. The users of the application were not given any instructions about how to use it or what to use it for. Data from the one-month study consisted primarily of interaction logs from the 42 participants. This was supplemented with phone interviews with four participants after the first and second week of use. All interviews were semi-structured using an interview guide, and recorded using a digital voice recorder.

\subsection{Data analysis}

The data from all three empirical approaches were analysed using grounded analysis. First, open coding was used to identify 393 different categories from the empirical data. These categories were then classified into 100 higher-level categories using axial coding. Finally, these categories were narrowed down to six themes using selective coding.

\section{Findings}

The data logs from the longitudinal field studies gave us a good overview of how and where the application was used as a part of the participants' everyday life. In total, the application was used 206 times, with usage frequency being higher at the beginning of the study, and decreasing towards the end. The logs showed that the Places Lens was used for the most time, but that the Public Transport Lens was used most interactively. Over the duration of the field study, 58\% of the usage time was spent in the Places Lens, 33\% in the Public Transport Lens, and 9\% in the People Lens. Looking at the number of times users interacted with content on the screen, $63 \%$ of this was done in the Public Transport Lens, $35 \%$ in the Places Lens, and $2 \%$ in the People Lens. The map view was active $70 \%$ of the time, whereas the AR view was active $30 \%$ of the time. The most 


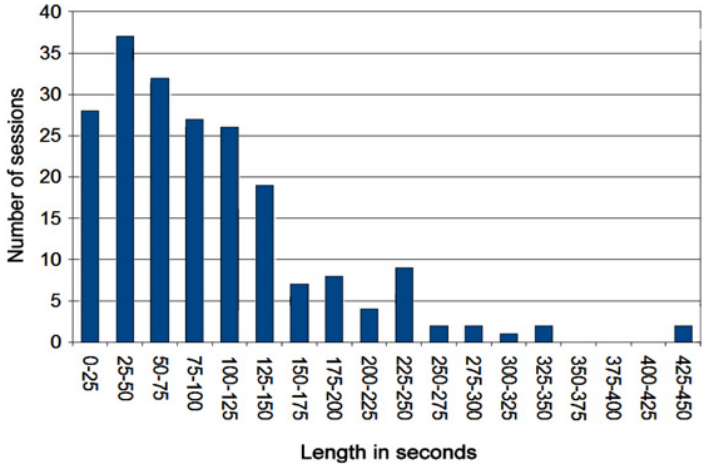

Fig. 7. Session lengths during the one-month study.

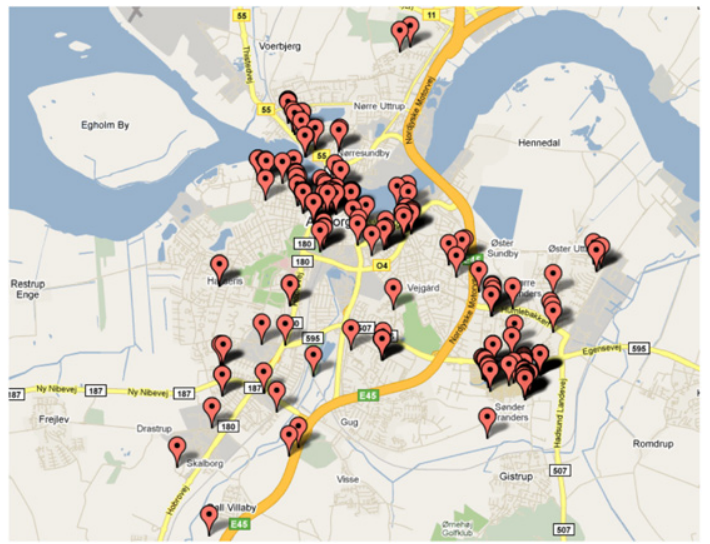

Fig. 8. Geographical distribution of use during the one-month field study.

common zoom level of the map view was the one zoomed out furthest, providing the most overview. Combining the logs of Lenses, ArcZooming, screen interaction, spatial movements, and map versus AR mode shows that context information in the Places Lens was explored more while moving around the city and by frequently changing between the map view and the AR view. Context information in the Public Transport Lens was used more while stationary and interacting with elements on the screen. The logs also showed that people used the application frequently but for short periods of time. The average duration of use was $100.8 \mathrm{~s}$, with a tendency towards less than $75 \mathrm{~s}$ (Fig. 7). This adds to the previous finding about mobile interactions happening with short bursts of attention [30] that the overall use of this type of mobile system happens in bursts of less than 2 min.

Looking at the geographical distribution of use (Fig. 8) shows two clusters around the city centre and the university campus, as well as scattered locations along the bus lines between those, and throughout the city's residential areas. This shows that the application was used in a variety of urban environments but mostly in physically dense or socially lively areas. From the log data it could be seen that the Places Lens was used mostly within the city centre, while at the university campus users were mostly using the Public Transport Lens.

\subsection{Feedback from long-term use}

The qualitative interviews complemented the data logs with details about the users' perception and actual use of the system in context. From these interviews, the most interesting insights came from the participants who used the system over longer periods of time as a part of their normal everyday life (i.e. the one-week and one-month studies).

The long-term users said that the system added a new dimension to the city, which they perceived in natural interplay with the other elements of the urban environment, and which contributed to their experience of the city as a whole. They used the system because it enhanced their experience of the environment around them in relation to what they were doing at the time, and not as much because it provided a clear-cut functional value. This manifested itself in the way they used the different Lenses and perceived their value, and in the way they reflected on the relative value of the map and augmented reality view. 


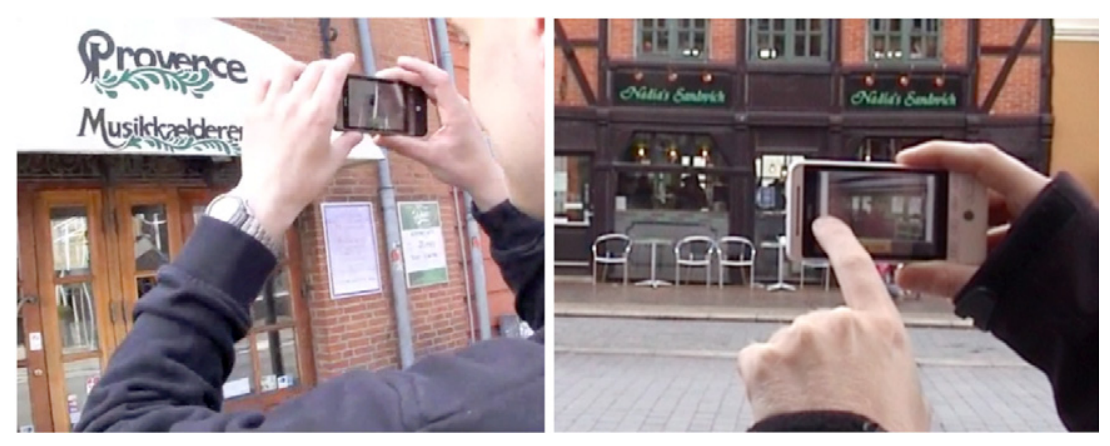

Fig. 9. Exploring physical context using the Places Lens.
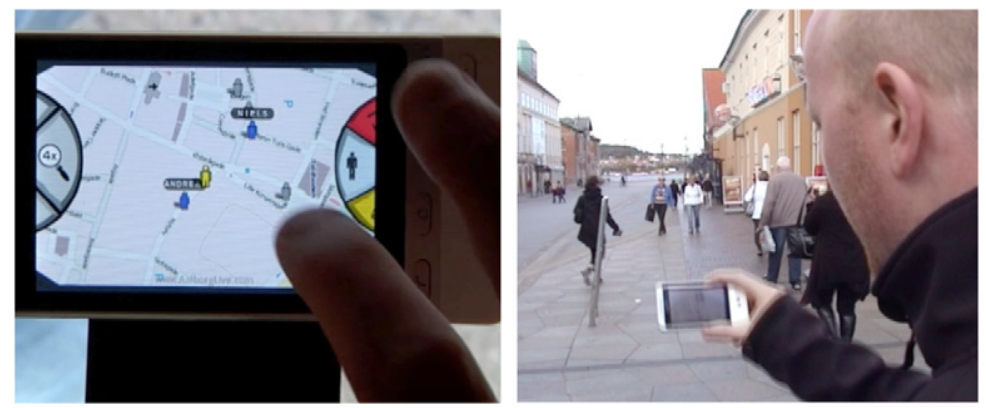

Fig. 10. Exploring social context using the People Lens.

\subsubsection{Places}

The Places Lens reportedly gave people a feel for what was located and going on behind the sometimes unapproachable and closed building facades around them, and a feel for the different districts of the city centre beyond their current street. In the map view, it gave streets and plazas, standing out as, for example, areas for dining or as areas for partying, a unique atmosphere that added to the ambience given off by these streets in their physical and social incarnation. In the AR view, the Places Lens brought the static elements of the city to life by adding a dynamic and interactive new dimension or sphere to the experience of facades, light, and sounds around the users. This ambient quality of the system meant that people used it for spontaneously exploring their surrounding context by interactively tuning into the different sources of content available around them. Because the digital contextual information provided was so closely related to the physical context, this reportedly created an experience of the situation as a whole, and of the city being responsive and interactive (see Fig. 9).

\subsubsection{People}

The People Lens reportedly contributed to the experience of the city, and the Places Lens, by creating an ambient sense of pulse and life to the urban experience as a whole. The representation of social context information in the People Lens showed that the city was alive and active, and that other people were also taking part of the digital experience of it. This reportedly added to the experience of social awareness about the surrounding environment and the feeling that the city was an inhabited and dynamic place. Most of the context information in the People Lens was purposely made ambiguous and incomplete by means of anonymization and lack of detail in order to overcome some of the issues of privacy related to revealing one's location in real time, and to see what meaning users would attach to such representations (see Fig. 10).

The user reactions to this were encouraging in the sense that people still made quite rich interpretations of social context. Clusters of anonymous people were perceived as groups gathering, and interpreted as an indicator of social activity in that area of the city. Similarly, seeing several of one's friends in the vicinity of each other was perceived as a grouping, and interpreted as a social event the user was missing out on, as observed directly in one of the one-hour studies: "Oh, there is Kai, and there is Andrea with him. They are hanging out over there without me!". Over the course of the study, however, some participants stopped using the People Lens. When asked about this in the interviews, they explained that either they had realized that this part of the system was partly simulated, or they felt that there was not often enough a critical mass of other people represented to create "a sustained reflection of the social activity in the city".

\subsubsection{Public Transport}

The Public Transport Lens was reportedly most popular because of its precise and detailed representation of a dynamic part of the user's context that would otherwise be invisible and difficult to grasp. The fact that this Lens presented people with live data reflecting something active happening in the city around them was highly appraised, both from a functional 


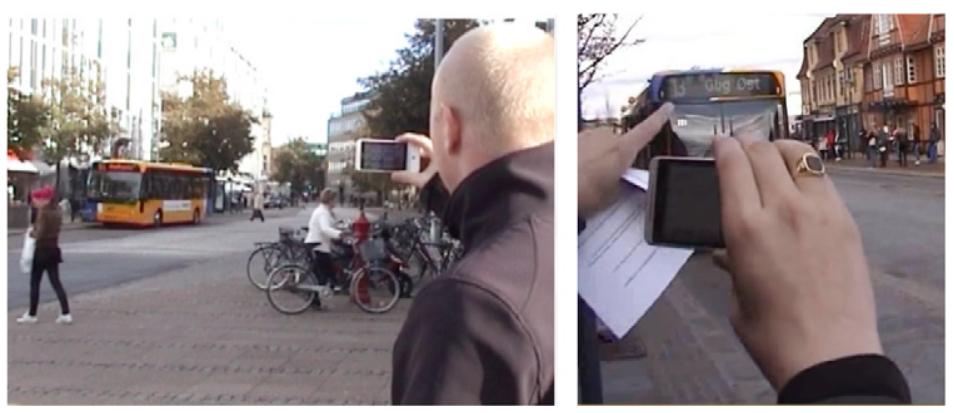

Fig. 11. Exploring temporal context using the Public Transport Lens.

point of view and in terms of the overall perception of the system in use that it contributed to. Hence, the Public Transport Lens contributed to the user experience in two ways. First, it provided an up-to-date representation of temporal context relevant for people here and now when they are going somewhere in the city. Second, and perhaps most interestingly, the fact that the system presented the user with such highly fresh contextual representation reportedly made people perceive the whole mobile system as a live view of the urban environment around them-even though parts of it were considerably more static. As expressed in relation to the Places and People Lenses, this experience of the system as an integral part of the urban environment created an atmosphere of the city being alive, responsive, and interactive (see Fig. 11).

\subsubsection{Lenses, maps, and augmented reality}

The interaction design of eSpective ${ }_{2}$ was met with positive feedback from most users. Most notably, people were impressed with how well the augmented reality view worked and how easy and smooth it was to modify and shift between views just by changing the way they were holding the mobile phone and by simple touch gestures on the screen. Because of the seamless transitions between Lenses, map view, and AR view, users spoke of the system as a whole rather than as a collection of disjoint views and functions. They expressed that it was "fun to play with" in itself, and that using it around the city enabled a playful exploration of their surrounding environment. In that sense, we found that the system created a ludic experience [31] that added to the overall immersive experience of the city, rather than a functional effective or efficient one disconnected from the urban environment around people.

\section{Discussion}

We have pursued a constructivist approach to context for context-awareness where context is an interpreted, socially constructed entity too complex to model in a computational system in its entirety, making only very simple context-based adaptation a feasible principle for mobile human-computer interaction. We believe that it is necessary to understand what value context-aware mobile systems offer to inhabitants and visitors in metropolitan areas. However, this value cannot optimally be described as one purely of functionality. Instead, we found that the value was highly qualitative and related to subjective factors such as playfulness, enchantment, and enjoyment experienced by the users, and, very importantly, experienced as an integral part of their experience of the urban environment around them. The role of eSpective 2 in people's life therefore did not appear to be as an information system for solving a particular set of tasks, but as a catalyst for living in, experiencing, and interacting with the city. This observation has led us to look beyond traditional HCI literature for discussing mediated context-awareness user experience and for analysing and describing people's life in and perception of metropolitan areas from a socio-architectural perspective.

\subsection{Urban ambience}

Urban ambience is defined as "the experienced physical and psychological qualities of the urban environment" [32,33], and is based on the belief that the experience of feelings such as comfort, satisfaction, and pleasure in a physical space is a result of people's interpretation and perception of the physical state of an architectural or urban space. According to Dupagne and Hégron [34], this is "the outcome of a complex composition of physical, physiological, psychological, sociological and cultural criteria", which arises from the interplay and relationships between factors such as building facades, vegetation, microclimate, light, soundscapes, human activities, and interactions, perceived within the overall frame of a person's own intentions and activities. This means that the notion of urban ambience offers a broad focus on the discussion of the intangible qualities of living and being in urban environments, also described by Alexander et al. as the "wholeness" [35], by Lynch as the "imageability" [36], and by Bentley et al. as the "responsiveness" [37] that characterizes a socially and culturally sustainable city [33].

According to Thibaud [1], an ambience-oriented approach puts emphasis on the enveloping nature of the world in which the perceiving subject is immersed, and it is ambience that links and unifies the many components of a situation or context. 
Thibaud [1] proposes three ways of characterizing urban ambience: (1) as the quality of the situation as a whole, (2) as a catalyst for action, and (3) as a sensory background. Ambience cannot be reduced to small individual parameters but relate to the whole contextual unity and it engages and involves us, engendering certain emotions and stimulating certain actions.

\subsection{Digital urban ambience}

We view digital urban ambience as the ambient feeling or experience in an urban environment created by digital technologies such as mobile phones and devices, situated displays, and other types of ubiquitous, pervasive, and mobile technologies. We found that the digital urban ambience manifested itself in several ways, and we apply Thibaud's three characteristics [1] in the sections below.

\subsubsection{The quality of the situation as a whole}

Our participants expressed how they experienced the digital information provided through the system as an integral part of their physical surroundings, enabling them to see things that could not be seen before. They described how they could look behind and beyond the built environment around them through the Places Lens, gauge the atmosphere given off by city districts as they appeared in their virtual incarnation through the Places and People Lenses, and how they could see dynamic elements of the city reflected through the Public Transport Lens. This new dimension or sphere reportedly added values such as life, responsiveness, and interactivity to a holistic experience of the city, and can as such be described as contributing to the quality of the situation as a whole. The physical, social, and temporal context information represented in the system all contributed to this quality and wholeness, albeit in different ways. The representation of physical context created additional sensations of the types, looks, and grouping of places, adding to the ambience created by physical facades, lights, and sounds; the representation of social context provided additional sensations of the inhabited city, adding to the urban ambience created by human activities and interactions; and the representation of temporal context provided additional sensations of the pulse of the city.

\subsubsection{A catalyst for action}

Our participants further expressed how aspects of context in the system triggered emotional responses and inspired, motivated, or drew them to go to certain places and do certain unplanned things. The representations of context reportedly engaged them in the world around them, brought out emotional responses, and prompted actions accordingly. The Places Lens revealed unknown or forgotten restaurants and bars that the user then visited or returned to. The People Lens revealed friends gathering without them, who they would then approach and join. The Public Transport Lens revealed an opportunity to get on a bus into town in $2 \mathrm{~min}$, which the user then ran for. The mediated view of context hence functioned as a mechanism for revealing affordances [38] of the surrounding environment as a set of resources for choices of activity, and can as such be described as a catalyst for action. Again, the different types of context information in the system all contributed to this aspect of the digital urban ambience created, but in different ways, by revealing affordances of a physical, social, or temporal nature. Representing other dimensions of context would, supposedly, influence the digital urban ambience with further or other catalysts for action.

\subsubsection{A sensory background}

Most participants did not articulate perceiving "ambience" as an individual measurable entity, but expressed instead their perception of the environment, or parts thereof, within the frame of certain moods, atmospheres, or ambiences. Hence, ambience was not something tangible that people referred to in the system or in the context, but something transcendent in the background. It was created and affected by elements in the context, as well as in the system, and at the same time recursively constituted a backdrop for people's sensations and experiences of these elements. The venues in the Places Lens, the individuals in the People Lens, and the busses in the Public Transport Lens were not perceived as "ambience" in themselves. But their collective presence in the different context Lenses in the system created a sensory background against which they were individually perceived and interpreted. Extending on from this line of thought, one potential way of greatly enhancing a future prototype system's ability to create a sensory background would be to network the individual devices and then capture and share the interpretation and mediation of context done by other users back into the system itself.

\section{Conclusions}

In this paper, we have presented our findings on the user experience of a mediated context-aware mobile system in an urban environment. We have argued that a new class of context-aware mobile systems is emerging enabled by the technical capabilities of modern mobile phones and operating systems such as iOS and Android OS. The interaction design of these systems explores a constructivism-based rather than a realism-based approach to context, which results in context-aware systems better described as mediated than as adaptive. As this is a new class of mobile systems, the body of knowledge about their use and user experience is still limited. In response to this, we have presented a specific example of a mediated contextaware mobile system for an urban environment, eSpective ${ }_{2}$, and a longitudinal field study of its use with a significant cohort 
of users. From our findings, we have argued that the value of mediated context-aware mobile systems is not optimally described as one purely of functionality, and that their user experience is not given full justice if only described by means of traditional usability-type metrics. Drawing on sociology and architecture, we have instead suggested that the user experience of such mediated context-aware systems in urban environments is conceptually described as one of digital urban ambience. Illustrating this concept, we have illustrated and discussed how context resources provided through eSpective ${ }_{2}$ digitally contributed to the experience or feeling of urban ambience by (1) adding to the quality of the situation as a whole, (2) constituting a catalyst for action, and (3) providing a sensory background for the users' experience of elements in the city around them.

For further research, we hope that this work will inform the constructivism-based approach to context, and help shape mediated context-aware mobile systems.

\section{Acknowledgments}

The authors thank the 58 participants in the field studies for their invaluable contribution to our findings. We also thank the anonymous reviewers of the paper for their positive feedback, and for interesting ideas on how to expand on the thinking about digital urban ambience.

\section{References}

[1] J.P. Thibaud, From situated perception to urban ambiences, in: Proc. 1st International Workshop on Architectural and Urban Ambient Environment, L'École d'Architecture de Nantes, 2002, pp. 1-11.

[2] Loopt. http://www.loopt.com, 2010.

[3] Layar. http://www.layar.com/, 2010.

[4] OneBusAway. http://onebusaway.org/, 2010

[5] B. Schilit, M. Theimer, Disseminating active map information to mobile hosts, IEEE Network 88 (1994) 22-32.

[6] R. McCall, I. Wagner, K. Kuutti, G. Jacucci, W. Broll, Urban mixed realities: technologies, theories and frontiers, in: Ext. Abstracts CHI 2008, ACM Press, 2008, pp. 3973-3976.

[7] P. Dourish, What we talk about when we talk about context, Personal and Ubiquitous Computing 8 (1) (2004) 19-30

[8] A. Oulasvirta, S. Tamminen, K. Höök, Comparing two approaches to context: realism and constructivism, in: Proc. CC'05, ACM Press, 2005 , pp. 195-198.

[9] M. Weiser, The computer for the 21st century-scientific American special issue on communications, Computers and Networks (1991).

[10] G. Chen, D. Kotz, A survey of context-aware mobile computing research, Paper TR2000-381, Department of Computer Science, Dartmouth College, 2000.

[11] B. Brown, R. Randell, Building a context-sensitive telephone: some hopes and pitfalls for context sensitive computing, Computer Supported Cooperative Work 13 (2004) 329-345.

[12] D. Dearman, K.M. Inkpen, K.N. Truong, Mobile map interactions during a rendezvous: exploring the implications of automation, Personal Ubiquitous Computing 14 (1) (2010) 1-13.

[13] E. Paulos, E. Goodman, The familiar stranger: anxiety, comfort and play in public places, in: Proc. CHI 2004, ACM Press, 2004, pp. 223-230.

[14] T. Erickson, Social translucence: designing social infrastructures that make collective activity visible, Communications of the ACM 45 (4) (2002) 40-44.

[15] B. Ferris, K. Watkins, A. Borning, OneBusAway: results from providing real-time arrival information for public transit, in: Proc. CHI 2010, ACM Press, 2010, pp. $1807-1816$.

[16] S. Lemmelä, H.J. Korhonen, Finding communication hot spots of location-based postings, in: Ext. Abstracts CHI 2007, ACM Press, 2007, pp. 2549-2554.

[17] A. Aaltonen, J. Lehikoinen, Refining visualization reference model for context information, Personal and Ubiquitous Computing 9(2005) 381-394.

[18] A. Dey, J. Mankoff, Designing mediation for context-aware applications, ACM Transactions on Computer-Human Interaction 12 (1) (2005) 53-80.

[19] W.K. Edwards, Putting computing in context: an infrastructure to support extensible context-enhanced collaborative applications, ACM TOCHI 12 (4) (2005) 446-474.

[20] J. Paay, J. Kjeldskov, Understanding the user experience of location based services: five principles of perceptual organization applied, Journal of Location-Based Services 2 (4) (2008) 267-286.

[21] R. Fithian, G. Iachello, J. Moghazy, Z. Pousman, J. Stasko, The design and evaluation of a mobile location-aware handheld event planner, in: Proc. Mobile HCI 2003, in: LNCS, Springer-Verlag, 2003, pp. 145-160.

[22] C. Zhou, P. Ludford, D. Frankowski, L. Terveen, An experiment in discovering personally meaningful places from location data, in: Ext. Abstracts CHI 2005, ACM Press, 2005, pp. 2029-2032.

[23] J. Paay, J. Kjeldskov, S. Howard, B. Dave, Out on the town: a socio-physical approach to the design of a context-aware urban guide, ACM TOCHI 16 (2) (2009) 1-34.

[24] A. Morrison, A. Oulasvirta, P. Peltonen, S. Lemmela, G. Jacucci, G. Reitmayr, J. Näsänen, A. Juustila, Like bees around the hive: a comparative study of a mobile augmented reality map, in: Proc. CHI 2009, ACM Press, 2009, pp. 1889-1898.

[25] J. Kjeldskov, J. Paay, e-Spective: pervasive computing presenting a new perspective of the city, in: Proceedings of the 17th Australasian Conference on Information Systems, Australasian Association for Information Systems, 2006.

[26] H. Hutchinson, W. Mackay, B. Westerlund, B.B. Bederson, A. Druin, C. Plaisant, M. Beaudouin-Lafon, S. Conversy, H. Evans, H. Hansen, N. Roussel, B. Eiderbäck, Technology probes: inspiring design for and with families, in: Proc. CHI 2003, ACM Press, 2003, pp. 17-24.

[27] OpenStreetMap. http://openstreetmap.org/, 2010.

[28] R. Bogdan, S.K. Biklen, Qualitative Research for Education: An Introduction to Theories and Methods, International ed., Pearson, 2006.

[29] N.K. Denzin, A Sourcebook: Sociological Methods, McGraw-Hill Inc., 1978.

[30] A. Oulasvirta, S. Tamminen, V. Roto, J. Kuorelahti, Interaction in 4-s bursts: the fragmented nature of attentional resources in mobile HCI, in: Proc. CHI 2005, ACM Press, 2005, pp. 919-928.

[31] Q. Stevens, The Ludic City: Exploring the Potential of Public Spaces, Routledge, London, 2007.

[32] T. Caplow, S. Stryker, S.E. Wallace, The Urban Ambience: A Study of San Juan, Puerto Rico, The Bedminster Press, Totowa, New Jersey, 1964.

[33] P. Osmond, Quantifying the qualitative: an evaluation of urban ambience, in: Proc. 6th International Space Syntax Symposium, $2007, \mathrm{pp} .1-7$.

[34] A. Dupagne, G. Hégron, Introduction, in: Proc. 1st International Workshop on Architectural and Urban Ambient Environment, L'École d'Architecture de Nantes, 2002.

[35] C. Alexander, S. Ishikawa, M. Silverstein, A Pattern Language, Oxford University Press, New York, 1977.

[36] K. Lynch, The Image of the City, MIT Press, 1960.

[37] I. Bentley, A. Alcock, P. Murrain, S. McGlynn, G. Smith, Responsive Environments, Butterworth Architecture, 1985.

[38] J. Gibson, The Ecological Approach to Visual Perception, Lawrence Erlbaum Associates, New Jersey, 1979. 